\title{
Government report critical of FDA blasted as misleading
}

Industry has roundly rebuffed a government report released at the end of last year that harshly criticized the US Food and Drug Administration (FDA) for its lax oversight of clinical trials. Many feel that the report not only criticizes the drug agency unfairly but also misrepresents FDA resources and staffing as currently adequate to carry out its rapidly expanding responsibilities-a conclusion clearly contradicted by a report from the FDA's Science Board released in late November.

The report, 'Food and Drug Administration's Oversight of Clinical Trials', issued by the Office of Inspector General (OIG) in the Department of Health and Human Services, which was widely reported in the lay media, came at the inopportune time of renewal of the Prescription Drug User Fee Act (PDUFA), with the agency in danger of closing and FDA staffers' jobs on the line.

Although the reauthorization of PDUFA under the FDA Amendments Act (FDAAA) of 2007 helped calm the atmosphere at the agency (Nat. Biotechnol. 25, 1189-1190, 2007), now some of the FDA's usual critics are coming to its defense.

Prompted by a congressional request, the OIG report focused on onsite bioresearch monitoring (BiMo) done under the auspices of the FDA Office of Regulatory Affairs during a five-year period ending with fiscal year 2005. In that period, FDA inspected only about $1 \%$ of such trial sites, according to the report, which also states that the agency's "ability to manage the BiMo program" is hindered by "data limitations" and "other factors." To help rectify this, OIG recommended the FDA develop better databases and registries, provide better feedback to BiMo inspectors and seek legal authority, which it lacks, for such oversight.

But several major criticisms of FDA in the OIG report are misleading at best, says Sara Radcliffe, who is vice president of Science and Regulatory Affairs for the Washington, DCbased Biotechnology Industry Organization (BIO). The $1 \%$ figure, for example, alludes to BiMo inspections that "account for only a tiny part of what FDA does to assure protection to participants in clinical trials," she says. "One could argue it's not enough, but it's never going to be $100 \%$," because that would waste resources. "Besides, FDA is not in the business of discovering problems after they occur, but in preventing them," she says.

Not only is "the percent of clinical sites being inspected misleading," says another critic of the report, who is familiar with BiMo through consulting with industry and universities on clinical trial design and who asked for anonymity, but "the method used for computing it" is also flawed. BiMo's inspections have an impact beyond just that $1 \%$, she says, because of the attention paid by the rest of the companies running clinical trials, particularly when faults are found with trials being conducted by prominent institutions. Another recommendation in the report-for "improved feedback" to onsite inspectors from top FDA officials-is "very touchy" and could be "problematic," she adds, because it could open the program to greater influence from political appointees.

Also at issue with BiMo, according to the OIG report, are agency shortcomings in tracking clinical trials and data. However, that should be getting better, as the FDAAA mandates improving such activities. Radcliffe says that FDA "is trying to build better clinical trial databases," but adds that the "universe" of the FDA is trials meant to support approvals anyway. "It's not the responsibility for FDA to assure protections for participants in other types of clinical trials," she says.

Alan Goldhammer, deputy vice president of regulatory affairs at the Pharmaceutical Research and Manufacturers of America (PhRMA) in Washington, says the report overlooks the large part individual firms play in monitoring clinical trials. "Companies want to make sure they're in compliance, so that when FDA does inspections there are no particular problems," he says, adding, "We've not heard that the monitoring activity of BiMo represents a drag on clinical trials. BiMo monitors for data quality and the integrity of institutional review boards. The real issue [in clinical trials] is 'Do the data meet endpoint requirements?"”

A biotech company regulatory affairs official, who also asked not to be identified, says that the industry is responsible for monitoring clinical trials sites, and "only if it's done poorly should FDA BiMo pick up the activity." In that regard, she says BiMo is really a matter of quality assurance- "the FDA checking to see that clinical trials are conducted according to protocol" and that data are accurate.
In terms of the clinical trials enterprise as a whole, "it's not as if FDA can tell all the academic centers and companies what to do," says BIO's Radcliffe. It simply wasn't built that way. Meanwhile, organizing those efforts and developing uniform standards for data collection and reporting are seen as desirable goals, and there are several new efforts to address those challenges. For instance, the Clinical Data Interchange Standards Consortium in Austin, Texas, is "doing tremendous work" leading one such effort, she says.

There's more help on the way. The FDA and Duke University Medical Center in Durham, North Carolina, late in November announced a joint effort to modernize clinical trials. The clinical research enterprise "needs to be much more streamlined and efficient, and at the same time it needs to be better equipped to answer the pressing questions that confront both patients and healthcare professionals," says FDA deputy commissioner Janet Woodcock. The partnership, which will be cochaired by Robert M. Califf, vice chancellor for clinical research at Duke, and Rachel Behrman, director of the FDA Office of Critical Path Programs, will consider developing national standards and technical improvements for streamlining the handling of clinical data and also will consider changing institutional review boards to minimize duplication of efforts during multicenter trials.

But promises to streamline data management and develop other technical improvements for managing clinical trials are of no use without the FDA having adequate resources. For example, the FDA Science Board, an advisory group to the agency, issued a blistering report in November, noting that the agency's "scientific base is badly eroded," its "scientific workforce does not have sufficient capacity and capability," and the agency "cannot fulfill its mission because its information technology system is inadequate." The main reason for these mounting problems, the science board maintains, is that FDA is stuck with "decreasing resources in the face of increasing responsibilities."

Put simply, says Radcliffe of BIO, "There are plenty of things FDA can do internally. They have the plans. They just need the money."

Jeffrey L. Fox, Washington, DC 